\title{
Crohn's Disease and Diagnostic Difficulty: A Case Report
}

\author{
*A Saeed ${ }^{1}$, A Shahin ${ }^{2}$, SC Dhar ${ }^{3}$, MMR Siddiqui $^{4}$, A Rahman ${ }^{5}$
}

\begin{abstract}
Crohn's disease is an uncommon disease in Bangladesh. Recently its prevalence is increasing. In the era of tuberculosis, it is sometimes difficult to diagnose in this subcontinent. Mrs. Bilkis Begum 50 years old housewife presented with loose motion with abdominal pain. She had significant weight loss. She was ill looking and anemic. Colonoscopy reveals multiple longitudinal ulcers in the descending colon. Biopsy finding revealed large number of granuloma with aggregates of epithelioid cells, histocytes and few giant cells. No caseation was found. In this scenario anti TB was started and condition of the patient was improved. She has gained her weight. After 6 month of anti Tb therapy repeat colonoscopy was done and revealed multiple healed longitudinal scar marks in the colon. In $15^{\text {th }}$ February 2018 she presented with previous complain. During this time her stool for calprotectin is high. Colonoscopy shows multiple longitudinal ulcers in the sigmoid colon and biopsy shows epithelioid cells and granulation. Now she was diagnosed as a case of crohn's colitis. Induction of therapy was started with prednisolone along with mesalamine. Condition of the patient improved.
\end{abstract}

Key Words: Crohn's disease, tuberculosis, colonoscopy, colitis.

\section{Introduction}

Crohn's disease, also referred to as regional enteritis, granulomatous enterocolitis, and terminal ileitis, is a chronic relapsing and remitting inflammatory disease of unknown cause that is often multifocal and can affect any portion of the gastrointestinal tract. ${ }^{1}$ It is generally accepted that the Scottish surgeon Dalziel gave the first account of the disease in $1913 .{ }^{2}$ In the 1960s LockhartMummery and Morson described the involvement of the large intestine by Crohn's disease 3 and it was in the 1950s that anal and anorectal Crohn's disease were described. ${ }^{4}$ In time it became clear that Crohn's disease can involve any part of the digestive tract and that extraintestinal manifestations can be present, especially in the skin, eyes and joints 5-9. We discuss, through the use of a case report features of Crohn's disease involving both the large intestine anal canal in a patient.

\section{Case Presentation}

Mrs. B Begum 50 years from Brahmanbaria was presented with loose motion ${ }^{8-9}$ times per day with abdominal pain. She lost her weight and felt weak. Examination reveals she is ill looking and anaemic.in May 2016 her $\mathrm{Hb}-10 \mathrm{gm} / \mathrm{dl}$, TLC$16.50 \times 10^{9} / 1$, ESR-85 mmhg, s. ALT-124, s.creatinine-.67 $\mathrm{mg} / \mathrm{dl}, \mathrm{N}-85 \%$, RBS-5.6 $\mathrm{mmol} / 1, \mathrm{CRP}-24.3 \mathrm{mg} / \mathrm{l}$, x-ray chest-normal, MTnegative, Colonoscopy reveals multiple longitudinal ulcers $80 \mathrm{~cm}$ from anal verge. Biopsy shows large

\footnotetext{
$1 *$ Dr. Atia Saeed, Assistant Professor, Department of Gastroenterology, Anwer Khan Modern Medical College, Dhaka

${ }^{2}$ Dr. Abu Shahin, Associate Professor, Department of Rheumatology, BSMMU

${ }^{3}$ Prof. Swapan Chandra Dhar, Professor \& Head, Department of Gastroenterology, Anwer Khan Modern Medical College, Dhaka

${ }^{4}$ Dr. Md. Mahmudur Rahman Siddiqui, Associate Professor, Department of Medicine, Anwer Khan Modern Medical College, Dhaka

${ }^{5}$ Dr. Anisur Rahman, Assistant Professor, Department of Gastroenterology, Sher E Bangla Medical College Hospital, Barishal

*Corresponding author
}

Date of submission: 12.04.2018 Date of acceptance: 25.05.2018

AKMMC J 2018; 9(2) : 154-157 
number of granuloma with aggregates of epithelioid histocytes and few giant cells. No caseation was found. At the same time endoscopy of upper GIT shows gastric ulcer. In this scenario anti TB was started and condition of the patient improved. She gained her weight.

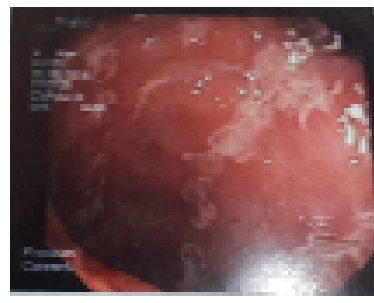

Fig-1: multiple longitudinal ulcers in the colon

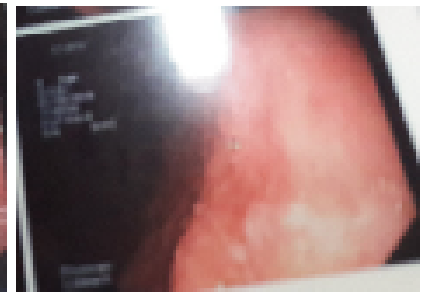

Fig-2: gastric ulcer in the body of stomach
After completion of 6 month her repeat colonoscopy reveals multiple healed longitudinal scar marks in the colon.

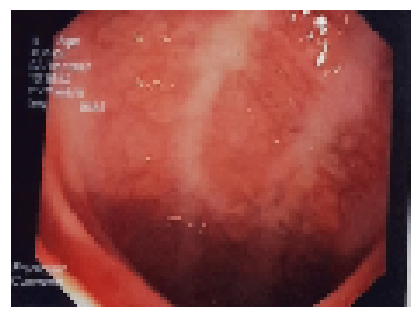

Fig-3: healed scar marks in the colon

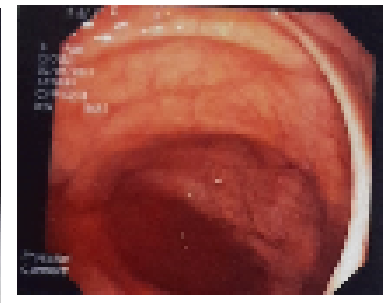

Fig-4: normal colon
In $15^{\text {th }}$ February 2018, Patient again presented with loose motion, abdominal pain, weight loss. At this time investigation reveals $\mathrm{Hb}-10 \mathrm{gm} / \mathrm{dl}$, ESR- 45 $\mathrm{mmHg}$, TLC-9x109/1, CRP- $34 \mathrm{mg} / 1$, stool for calprotectin-653 $\mu \mathrm{g} / \mathrm{gm}$. colonoscopy shows multiple longitudinal rake like ulcers with mucosal swelling $35 \mathrm{~cm}$ from anal verge. Fistula with pus seen in the perianal region. Biopsy reveals epithelioid cells and granulation. Now she was diagnosed as a case of crohn's colitis. Induction therapy started with prednisolone $40 \mathrm{mg}$ /day along with mesalamine. symptoms subside and condition of the patient improved. Now she is on maintenance therapy and on regular follow up.

\section{Discussion}

The prevalence of Crohn's disease has been difficult to estimate because of the errors regarding the distinction between large intestinal Crohn's disease and ulcerative colitis. ${ }^{10}$ Crohn's disease has a slightly higher incidence in females, and occurs at all ages. ${ }^{1}$ It is considered an uncommon disease, with an estimated incidence of 5 to 20 cases per 100.000 population annually. ${ }^{1}$ There are two peaks of incidence, one at 20 to 30 years of age, and the other in the 60-70 age group. ${ }^{1,10}$ Familial association is found in approximately $10 \%$ of the cases. ${ }^{1}$ The etiology of Crohn's disease remains completely unknown, although several theories have been issued, such as the involvement of genetic factors, environmental factors (including diet), and infective agents. ${ }^{10}$ Concerning clinical features, the initial presentation may be indolent or may be acute and severe. ${ }^{1}$ The symptoms are variable but often include cramping pain, typically localized to the right lower quadrant, nonbloody diarrhea, as well as fever, malaise, and anorexia. ${ }^{1}$ Hemorrhage and hematochezia are uncommon, but patients may present iron deficiency anemia due to occult gastrointestinal bleeding. ${ }^{1}$ Extraintestinal manifestations, when present, involve inflammatory changes in joints, eyes, liver, and skin1. Because resection is typically performed only in patients who have severe complications, the gross pathologic findings will be often significant. ${ }^{1}$ The subserosal fat is often firm and contracted over the areas of involvement. ${ }^{1}$ Longitudinal opening of the bowel may reveal areas with normal bowel wall thickness and other areas of the bowel that are firm, thickened

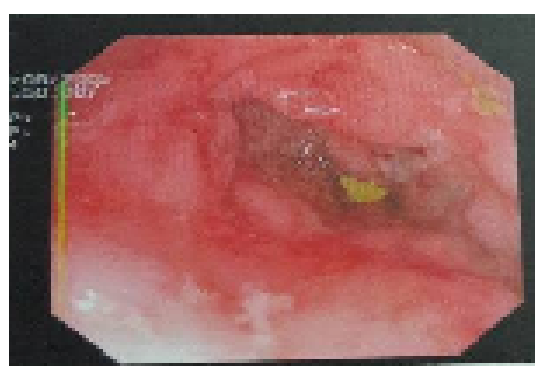

Fig-5: longitudinal ulcers in sigmoid colon

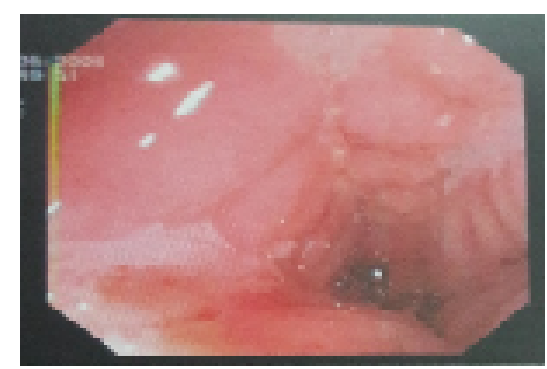

Fig-6: mucosal swelling along with ulcer

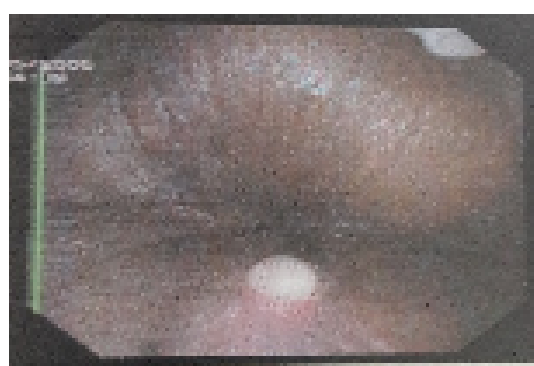

Fig-7: anal abess 
and pipelike. ${ }^{1}$ Interloop adhesions occur. ${ }^{1}$ The mucosa may show a variety of changes, including aphthous erosions, longitudinal "rake" ulcers, cobblestoning, polyps, fissures, and fistulas. ${ }^{1}$ The process is usually multifocal, often with rectal sparing, but may become confluent. ${ }^{1}$ When confluent, the firm pipelike nature of the bowel may help to distinguish Crohn's disease from ulcerative colitis, where fibrosis is unusual. ${ }^{1}$ The most characteristic microscopic features of the disease are its multifocal involvement, focal ulceration and transmural inflammation in the form of lymphoid aggregates and granulomas. ${ }^{10}$ It is important to know that granulomas may be absent or poorly formed. ${ }^{1,11}$ When found, ulcers are typically longitudinally oriented, separated by histologically normal edematous mucosa. ${ }^{1}$ When granulomas and fissuring ulcerations are absent, the most prevalent and diagnostically useful feature is transmural inflammation in the form of multiple lymphoid aggregates, scattered throughout the bowel wall but particularly obvious in the submucosa and subserosa10. No specific tests for Crohn's colitis are available. In cases in which distinguishing between ulcerative colitis and Crohn's colitis is difficult, performing both ASCA(antiSaccharomyces cerevisiae antibody) and P-ANCA tests may help in the differential diagnosis because P-ANCA test results are positive in approximately $70 \%$ of patients with ulcerative colitis and in $10 \%$ to $30 \%$ of patients with Crohn's disease. ${ }^{1}$ The overlap of results between Crohn's disease and ulcerative colitis impose further research, thus underlining the importance of a histopathological confirmation. ${ }^{1}$ Fulminant Crohn's colitis can be difficult or impossible to distinguish from ulcerative colitis in a mucosal biopsy specimen; furthermore, in fulminant (toxic) ulcerative colitis, inflammation may become transmural. ${ }^{1}$ The effects of treatment can also cause diagnostic difficulty because treated ulcerative colitis may show areas of involved and uninvolved mucosa and variable degrees of activity. ${ }^{1}$ Crohn's colitis must be also distinguished from infectious colitis and medication-associated colitis; histologically, the erosions of Crohn's colitis are associated with a chronic inflammatory infiltrate, while in infectious colitis is characterized by a predominance of neutrophils rather than lymphocytes and plasma cells. ${ }^{1}$ Medication-induced erosions usually have negligible acute inflammation, and typically only a few neutrophils are seen in the area of erosion. ${ }^{1}$ Another important differential diagnosis of Crohn's disease is tuberculosis. Histological features favoring a diagnosis of tuberculosis include the number, size and coalescence of granulomas and the presence of caseating necrosis. ${ }^{12}$ Transmural inflammation in the form of lymphoid aggregates, small granulomas, focal inflammation and poorly formed granulomas favor Crohn's disease. ${ }^{12}$ No cure for Crohn's disease was found. Treatment focuses on inducing and maintaining remission, minimizing side effects, and improving the quality of life. ${ }^{1}$ Standard therapy includes anti- inflammatory drugs, mainly 5-ASA (5-aminosalicylic acid) compounds as sulfasalazine and other salicylates, and steroids; infliximab, a monoclonal antibody against tumor necrosis factor?, has been found to be helpful in treating acute episodes of Crohn's colitis, particularly fistulas and other complications. ${ }^{1}$ A correct diagnosis in an early stage of the disease's evolution is ideal not only because it implies avoiding local and systemic complications, but also because of the increased risk for adenocarcinoma in patients with Crohn's disease. ${ }^{1}$ Multiple synchronous or metachronous adenocarcinomas may occur and involve the large and small intestines. ${ }^{1}$ Overall mortality for adenocarcinomas arising in Crohn's disease is high, approximately $80 \%$, mainly because of the similarities between symptoms of Crohn's disease relapse and symptoms of developing cancer, which may lead to a delayed diagnosis of the adenocarcinoma. ${ }^{1}$

\section{Conclusion}

Although considered by many an uncommon entity which affects mainly young adults, it is important to know that Crohn's disease may debut at any age. The symptoms for which patients may require medical attention can mimic a wide variety of disorders whose treatments greatly differ. Establishing an appropriate treatment in order to avoid short and long- term complications, which may be life-threatening to the patient, depends mainly on distinguishing between other inflammatory disorders of the digestive tract and Crohn's disease. A histopathologically confirmed diagnosis becomes greatly necessary also because of 
the increased risk of adenocarcinoma in patients with Crohn's disease.

Conflict of interest: We have no conflict of interest.

\section{References}

1. Iacobuzio-Donahue, C. A. \& Montgomery, E. A. (2012). Idiopathic inflammatory bowel disease. In J. R. Goldblum (series editor), Gastrointestinal and Liver Pathology. USA: Elsevier Saunders

2. Dalziel, T. K. Chronic interstitial enteritis. $\mathrm{Br}$ Med J, 2013; ii: 1068

3. Lockhart-Mummery, H. E. \& Morson, B. C. Crohn's disease (regional enteritis) of the large intestine and its distinction from ulcerative colitis. Gut. 1960; 1: 87

4. Morson, B.C. \& Lockhart-Mummery, H. E. Anal lesions in Crohn's disease. Lancet. 1959; ii: 1122

5. Fielding, J. F., Toye, D. K., Beton, D. K et al. Crohn's disease of the stomach and duodenum. Gut. 1970; 11: 1001

6. Basu, M. K., Asquith, P., Thompson, R. A. et al. Proceedings: Oral lesions in patients with Crohn's disease. Gut. 1974; 15: 346.

7. Huchzermeyer, H., Paul, F., Seifert, E., et al. Endoscopic results in five patients with Crohn's disease of the esophagus. Endoscopy.1977;
8. Rankin, G. B., Watts, H. D., Melnyk, et al. National Cooperative Crohn's Disease Study: extraintestinal manifestations of perianal complications. Gastroenterology. 1979; 77: 914

9. Greenstein, A. J., Sachar, D. B., Smith, H., et al. Patterns of neoplasia in Crohn's disease and ulcerative colitis. Cancer. 1978; 46: 403

10. Day, D. W., Jass, J. R., Price, A. B., Shepherd, N. A., Sloan, J. M., Talbot, I. C., Warren, B. F. \& Williams, G. T. (2003). Inflammatory disorders of the small intestine (pp. 288-298); Inflammatory disorders of the large intestine (pp. 504-511). In: Morson and Dawson Gastrointestinal Pathology. Blackwell Science, Blackwell Publishing Company.

11. Heimann, T.M., Miller, F., Martinelli, G., et al. Correlation of presence of granulomas with clinical and immunological variables in Crohn's disease. Arch Surg. 1988; 123: 46

12. Pulimood, A.B., Ramakrishna, B.S, Kurian, G. et al. Endoscopic mucosal biopsies are useful in distinguishing granulomatous colitis due to Crohn's disease from tuberculosis. Gut. 1999; 45:537 